\title{
SOPHISMUS UND SEINE BEURTEILUNG IM TALMUD
}

GEORG NÁDOR

Academia Maimonideana. London

1.

Julius Guttmanns Die Philosophie des Judentums (München 1933, und seitdem nachgedruckt) ist in vieler Hinsicht auch heute noch ausschlaggebend. Sein Neokantianismus hat aber in seinen Urteilen einige Einseitigkeiten verursacht. Dies geschah unter anderen in seiner Beurteilung der talmudischen Weisheit. Er behauptet kategorisch: das rabbinische Judentum war von der wissenschaftlichen Philosophie der Griechen nicht beeinflusst. Höchstens von den Popularisationen dieser Philosophie (Seite 50). Und unter den Richtungen, die in populärer Formen das talmudische Denken beeinflussten, erwähnt er Platon und die Stoa. Sophismus und Sokratik werden in dem umfassenden Buch nicht einmal aufgezählt.

Der Autor folgender Zeilen ist anderer Meinung. Wir glauben beweisen zu können, dass Sokratik und Sophistik im Talmud etlichemal zum Wort kommen, meist in verhüllter Form, ohne die Namen der Richtungen zu erwähnen. Man kann auch zeigen, dass die Sophistik bzw. die sophistische Denk- und Argumentationsmethode während der Jahrhunderte von den Talmudisten in verschiedener Weise beurteilt wurde, und die Werturteile darüber sich mehrmalig geändert hatten. Die Debatten über diese Frage wurden von den Rabbinern in einer 'Bildsprache' geführt. Die Terminologie dafür war erst später während des Mittelalters geprägt.

2.

Es gibt in der rabbinischen Literatur einen Terminus technicus für Sophismus: חריפות של הבל harifût šel hebel (falsche Spitzfindig- 
keit) '. Dieser Ausdruck wird auch in der späten rabbinischen Literatur behalten. Samuel Edels im 16-17 Jahrhundert, übernimmt ihn von den Tôsafót (13 Jahrhundert), und prägt dazu den begrifflichen Gegensatz: חריפות של אמת harîfût šel 'emet (wirklicher Scharfsinn) 2.

Das beste Beispiel für sophistische Denkweise im Talmud ist: ein Reptil für ein reines Tier zu erklären mit Hilfe von vielen Argumenten: לטהר את השרץ lè-țaher 'et ha-ŝeres. In diesem Fall ist "Reinwaschen des Reptils" bloss eine Allegorie. Schon Samuel Edels hat das richtig gesehen. Er kommentiert: der Talmud spricht davon, dass einer fähig ist für einen, der belastet ist, entlastende Argumente aufbringen ${ }^{3}$.

3.

Die Sophistik als philosophische und logische Richtung hat sich zuerst in Griechenland vor und während der Aktivität des Sokrates entwickelt. Die Sophisten haben die Kunst der Argumentation auf eine Höhe gebracht, die als Virtuosität gelten kann. Es wurde von den Vertretern dieser Richtung gesagt, dass sie sich damit prahlen: "Die schwache Sache (vor dem Gerichtshof) als die Starke erscheinen zu lassen" ${ }^{4}$.

Diese Richtung verbreitete sich vornämlich unter Rechtsanwälten und Rhetoren. Die sophistische Dialektik wurde in den Rhetorschulen - die mit der Zeit auch in den Provinzen Roms ins Leben kamengepflegt und für reifere Studenten angeboten. Viele gedankliche Elemente der Sophistik gingen auch in die skeptische PhilosophieRichtungen über, wo behauptet wurde, dass wegen der Vielseitigkeit der Phänomene niemand imstande ist ein objektiv gültiges Urteil zu fällen. Um 200 nach Christi hat die Sophistik in der griechischrömischen Kultur eine Renaissance erlebt. Dieser Umstand ist für unsere Untersuchung nicht unwichtig.

Auch innerhalb der rabbinischen Denkweise sieht man nämlich

1 Tosaphoth, 13 Jahrhundert, zu Sanhedrin 17/a. שיודע לטהר את השרץe-yôde`a lĕtaher 'et ha-seres".

${ }^{2}$ Hiddusê Maharscha zur Stelle in Talmud Babli Trakt. Sanhedrin.

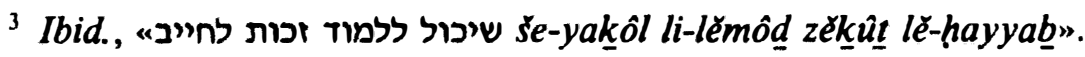

${ }^{4}$ Die Worte des Protagoras von Abdera. Siehe: H. DiEls, Die Fragmente der Sokratiker, Rowohlt 1963, S. 123. 
um diese Zeit einen wesentlichen Standpunkt-Wechsel in Sachen der Sophistik und der übermässigen Dialektik.

Dokumente aus dem zweiten Jahrhundert zeigen, dass man in jener Zeit denjenigen gegenüber vorsichtig war, die als Virtuose des Debattierens, ihren Standpunkt mit unzähligen Argumenten zu bekräftigen versuchten.

Der alte Gelehrte Dosa ben Arkinos nannte seinen Bruder «Teufelskerl», (בכור שטן běkôr Śțan), der für seinen problematischen Standpunkt «300 Argumente aufbringen kann» 5. Bei dieser Äusserung waren die Grössen des zweiten Jahrhunderts, R. Eliezer ben Hyrkanos, R. Eleazar ben Azarja, Rabbi Akiba, dabei gewesen und keiner von ihnen widersprach dieser Anschauung, die zur Vorsicht mahnte.

Eine andere Quelle aus dem zweiten Jahrhundert zeigt, dass Sokratik und auch Sophistik den Rabbinern bekannt war, und sie mit diesen Denkformen sogar gespielt haben, sie aber nicht ohne Kritik annahmen. "Wer sagt, er habe kein Wissen, hat bestimmt kein Wissen" (R. Jose).

Eine andere Version alternative vorgeschlagen: "Wer sagt, er habe nichts anderes als Wissen, hat bestimmt nichts anderes als Wissen". Darauf wird erwidert, dass dies einfach Trivialitäten sind. Eine dritte Version wird vorgeschlagen: "Wer sagt, er habe nichts anderes als Wissen, der hat nicht einmal Wissen" (Yëbamôt 109/b.).

Die erste Version erinnert uns an die Selbstcharakterisierung des Sokrates, die anderen an sophistische Thesen. In dieser Zeit waren die Rabbiner dieser Gedankenweise gegenüber kritisch eingestellt.

In kurzer Zeit änderte sich aber die allgemeine Meinung.

4.

Wann ist die Formel: «Das Reptil für rein zu deklarieren» entstanden? Spätere Quellen im Talmud wollen die Formel bis Jabne (70 n. Ch.), folgen. Zuverlässig dokumentieren lässt sich die Formel aber erst seit dem dritten Jahrhundert. In dieser Periode wird die als sophistisch scheinende Dialektik von grossen Meistern des Talmuds sehr warm angenommen. Man betont mit Nachdruck, dass rhetoris-

\footnotetext{
${ }^{5}$ Jeb. 15/a.
} 
che Gewandheit und dialektische Spitzfindigkeit Qualitäten sind, die ein angehender Richter unbedingt sich aneignen muss:

"Sagte R. Jehuda im Namen von Raw, (drittes Jahrhundert): man ernennt niemanden in den grossen Gerichtshof (Sanhedrin), der nicht fähig ist das Reptil mit Argumenten aus der Thora als rein zu erklären" ${ }^{6}$.

Die Frage der Sophismen wurde -laut dem palästinensischen Talmud - auch etwas später debattiert und das sophistische Denken wurde in einem noch positiveren Sinne empfohlen:

"Sagte R. Jochanan: wer unfähig ist das Reptil so beurteilen, dass er es hundertmal, d. h. mit hundert Argumenten, rein, bzw. unrein erklärt, der darf im Gerichthof nicht auftreten" ?.

Diese Position wird auch mit einer Anekdote bekräftigt:

"Moses bat Gott: Herr der Welt, ich bitte dich lass mich wissen wie die Gesetz-Entscheidungen (Halacha) ausfallen sollen. Antwortete ihm Gott: Richte nach der Mehrheit. Sind jene in der Mehrheit, die den Angeklagten für unschuldig halten, soll er unschuldig gelten. Sind jene in der Mehrheit, die den Angeklagten für schuldig erklären, soll er als schuldig betrachtet werden. Damit das Gesetz interpretiert werde auf folgende Art: mit 49 Argumenten ihn als "unschuldig" zeigend, respektive mit 49 Argumenten ihn als "schuldig" zeigend" 8 .

Die Dokumente zeigen genügend, dass während einer bestimmten Periode die damals international wieder sich erstärkende sophistische Methode von den Talmudisten gar nicht in Frage gestellt wurde. Im Gegenteil: sie sahen nur die Vorteile sowohl für die Rechtsentscheidungen, wie für fragliche Fälle der kultischen Reinheit.

Wie kann man aber eine Methode gebrauchen, die auf der Basis eines radikalen Relativismus steht? Die Rechtfertigung ist in der obigen Anekdote gegeben. Nicht die Wahrheit ist relativ. Die grosse Zahl der Argumente 'für' und 'gegen' eine Rechtsentscheidung zeigt bloss die Alternativen der möglichen Anschaungsweisen. Die Richter

\footnotetext{
${ }^{6}$ Sanh. 17/a.

7 Jer. Sanh. 22/a.

8 Ibid.
} 
können das auswählen, was sie überzeugend finden. Und das Urteil der Mehrheit entscheidet.

Somit wurden in der ersten Periode nach Bekanntschaft mit der Methode des Neo-Sophismus, die positiven Aspekte der Methode hervorgehoben. Bald kam aber auch die Kritik.

5.

Der locus classicus im Talmud für die Kritik der sophistischen Methode ist 'Erûbîn 13/b. Da sind verschiedene Dokumente gesammelt über dieses Problem aus der Periode nach 300. Man erfährt, dass mehr und mehr Stimmen gegen die Methoden der Sophistik hörbar werden, die Kritik wird sogar in frühere Zeiten zurückgesezt. Der Text fängt gerade mit einer 'Umwertung der Werte' an:

"Sagte R. Acha bar Chanina: es ist evident vor dem Schöpfer der Welt, dass in Rabbi Meirs Generation er der beste Gelehrte war. Und warum wurde die Halakâ nicht nach ihm (d. h. seinen Standpunkt gemäss) fixiert? Weil seine Kollegen seine Methode nicht verstehen konnten, mit der er das Unreine als rein erklärt hat, und dafür Gründe aufbrachte, und auch umgekehrt»?

R. Acha, der Tradent dieser Úberlieferung lebte 5-6 Generationen nach R. Meir. R. Meirs Ruhm war noch immer stark. Man fragte sich: schon R. Meir hat eine Mischna zusammengestellt, warum wurde sie aber nicht als die autoritative angenommen?

Die Tradition lehrt, dass die Ursache in R. Meirs Argumentationstechnik lag. Die Kollegen hätten diese Technik nicht verstanden, oder nicht gebilligt. Sie sollte die sophistische Methode gewesen sein: 'für' und 'gegen' eine und dieselbe Sache zu argumentieren.

Ob die Kritik der sophistischen Methode so weit in der Zeit zurückgeht ist fraglich. Wahrscheinlich kam die Methode erst 'nach' R. Meir in Verruf. Der Berichterstatter im Talmud gestaltet aber seine Geschichstradition nach den Wertmassstaben seiner eigenen Epoche.

Nach einem kleinen Umweg kehrt der Talmud ('Erûbîn) zum Problem der Sophistik zurück:

\footnotetext{
${ }^{9}$ Erub. 13/b.
} 
"Sagte R. Abahu im Namen R. Jochanan: Rabbi Meir hatte einen S h hüler, der hiess Symmachus. Er bekräftigte das Unreine mit 48 Argumenten der Unreinheit (טעמי טומאה tacame tûme’â) und jede reine Sache mit 48 Argumenten der Reinheit (טעמי טהרה tacamê tohorâ)" ${ }^{10}$.

R. Abahu meint, man muss die dialektische Argumentationstechnik nicht mit der Sophistik verwächseln. Man kann in der Dialektik exzellieren auch dann, wenn man das Reine mit vielen Argumenten als rein, das Unreine mit ebensoviel Argumenten als unrein beweist, und nicht miteinander verwechselt.

Der Talmudtext kommt dann wieder zum Problem der Sophistik zurück und erinnert an einen Fall aus der Vergangenheit, als ein jüngerer Gelehrte mit reiner Sophistik operiert hat:

"Wir haben gelernt: es gab einen hervorragenden Studenten in Jabne, der das Reptil mit 150 Argumenten als rein deklarierte. Rabina sagte dazu: ich könnte formell dasselbe machen. Das war bloss eine 'Geschichte' (Ohne prinzipielle Wichtigkeit)." ".

Diese Zusammenstellung des Materials über die Sophistik im Traktat Erubin: stammt aus einer relative späten Periode. Rabina, der dort als einer der Tradenten auftritt wirkte um 400 nach Christi. Er und R. Abahu nahmen Stellung gegen die reine Sophistik. Sophistik und Dialektik werden von ihnen begrifflich und praktisch auseinandergehalten.

Die späten Amoräer haben sich kategorisch gegen sophistische Denkmethoden gewandet. R. Assi, (gestorben 425), Leiter der Akademie in Sura, hat als äusserste Degradation des Denkens folgende Methode gehalten: das reine als unrein, das Unreine als rein zu deklarieren ${ }^{12}$.

Eine parodistische Darstellung der sophistischen Denkweise steht im Traktat Derek 'Eres aus einer späteren Zeit und lautet:

«Folgende Frage stellte R. Josse ben Taddai, ein Mann aus Tiberias Rabban Gamaliel: (Man könnte folgenderweise argumentieren). Es

\footnotetext{
${ }^{10}$ Ibid.

"In einer ähnlicher Version Sanh. 17/a, sagte das Raw, wahrscheinlich ist die richtige Lesart auch dort: Rabina.

12 Gen. Rabbd. 12/1.
} 
ist eine Tatsache, dass ich die Tochter meiner Frau nicht heiraten darf, obwohl ich meine Frau haben kann. Um wieviel mehr ist verboten die Tochter irgendeiner fremden verheirateten Frau zu ehelichen wo ich nicht einmal die Mutter heiraten darf" ${ }^{13}$.

\section{Die Sophistik - haben die späteren Rabbiner gemerkt - ist eine gefährliche Waffe ${ }^{14}$.}

\section{RESUMEN}

La visión general sobre el pensamiento talmúdico ha sido formulada enfáticamente por Julius Guttmann: los sabios talmúdicos no estaban influidos por la filosofía griega. Esta tesis debe ser revisada. Une de los casos cruciales en este punto es la actitud de los rabinos hacia los métodos de argumentación socráticos y sofisticos. El presente estudio muestra que, aunque la técnica de términos para el pensamiento escéptico no emergió hasta la Edad Media, el problema en sí mismo se remonta a los tiempos misnaicos. Se debatía bajo la cobertura de una imagen metafórica concreta: "purificar al reptil con mumerosos argumentos". Durante la época talmúdica se pueden distinguir tres períodos sucesivos en la valoración de esta forma de pensamiento: 1) una actitud negativa, hasta el año 200; 2) bajo la influencia de las corrientes neo- sofísticas, una evaluación positiva, especialmente desde el año 200 al $300 ; 3)$ una actitud crítica que hace distinción entre los métodos sofísticos y dialécticos.

\section{SUMMARY}

The general view about Talmudic thinking had been emphatically formulated by Julius Guttmann: the Talmudic sages were not influenced by Greek philosophy. This thesis must be re-examined. One of the crucial cases in this point is the attitude of the Rabbis towards Socratic and sophistical methods of argumentation. The present study shows that, although the terminus technique for sceptical thinking did not emerge in Rabbinical thought until the middle ages, the problem itself goes back to mishnaic times. It was debated under the cover of a concrete metaphorical image: "to purify the reptile with numerous arguments". Evaluating this way of thinking during the Talmudic epoch three successive periods can be distinguished: 1) a negative attitude up to $200 ; 2$ ) under the influence of neo-sophistical currents, a positive evaluation, especially from 200 till 300. 3) a critical attitude distinguishing between sophistical and dialectical methods.

${ }^{13}$ Derek 'Eres Rabbâ, Kap. 1.

14 Vielleicht war dieses Thema schon früh in der Talmudzeit als komisches Thema bearbeitet. Vgl. G. NADOR, Die jüdische Fabel im Altertum, Northwood 1994, S. $60 \mathrm{ff}$. 